\title{
Prior information: how to circumvent the standard joint-measurement uncertainty relation
}

\author{
Michael J. W. Hall \\ Theoretical Physics, IAS, \\ Australian National University, \\ Canberra ACT 0200, Australia
}

\begin{abstract}
The principle of complementarity is quantified in two ways: by a universal uncertainty relation valid for arbitrary joint estimates of any two observables from a given measurement setup, and by a general uncertainty relation valid for the optimal estimates of the same two observables when the state of the system prior to measurement is known. A formula is given for the optimal estimate of any given observable, based on arbitrary measurement data and prior information about the state of the system, which generalises and provides a more robust interpretation of previous formulas for "local expectations" and "weak values" of quantum observables. As an example, the canonical joint measurement of position $X$ and momentum $P$ corresponds to measuring the commuting operators $X_{J}=X+X^{\prime}, P_{J}=P-P^{\prime}$, where the primed variables refer to an auxilary system in a minimum-uncertainty state. It is well known that $\Delta X_{J} \Delta P_{J} \geq \hbar$. Here it is shown that given the same physical experimental setup, and knowledge of the system density operator prior to measurement, one can make improved joint estimates $X_{\text {opt }}$ and $P_{\text {opt }}$ of $X$ and $P$. These improved estimates are not only statistically closer to $X$ and $P$ : they satisfy $\Delta X_{\text {opt }} \Delta P_{\text {opt }} \geq \hbar / 4$, where equality can be achieved in certain cases. Thus one can do up to four times better than the standard lower bound (where the latter corresponds to the limit of no prior information). Other applications include the heterodyne detection of orthogonal quadratures of a single-mode optical field, and joint measurements based on Einstein-Podolsky-Rosen correlations.
\end{abstract}

PACS numbers: 03.65.Ta 


\section{INTRODUCTION}

At least four generic types of uncertainty principle can be distinguished in quantum theory:

(i) State preparation: the quantum description of a physical system cannot simultaneously assign definite values to all observables;

(ii) Overlap: different physical states cannot in general be unambiguously distinguished by measurement;

(iii) Disturbance: measurement of one observable necessarily "disturbs" other observables;

(iv) Complementarity: the experimental arrangements for accurately defining/measuring different observables are in general physically incompatible.

These principles are all negative in content, corresponding to limits on what is possible in quantum mechanics. These limits are quantified via associated uncertainty relations. As the literature on such uncertainty relations is extensive, only a few general remarks and indicative references will be given here to set the context for this paper.

The "state preparation" uncertainty principle is the best known, and places limitations on classical notions of prior knowledge (and hence predictability). The corresponding uncertainty relations are generally expressed in terms of the spreads of the probability distributions of different observables, the prototypical example being the textbook inequality

$$
\Delta X \Delta P \geq \hbar / 2
$$

for the rms spreads of position and momentum.

The "overlap" uncertainty principle corresponds to the existence of non-orthogonal states, and underlies the semi-classical notion that quantum states occupy a phase space area of at least $2 \pi \hbar$. It also separates quantum parameter estimation from its classical counterpart. A corresponding uncertainty relation is the parameter estimation bound

$$
\delta X \Delta P \geq \hbar / 2
$$

where $\delta X$ is a measure of the error in any (covariant) estimate of the amount by which a state has been displaced in position, and $\Delta P$ is the rms momentum spread of the state $[1,2,3]$.

The "disturbance" uncertainty principle is connected to early statements by Heisenberg such as 'every subsequent observation of the position will alter the momentum by an un- 
known and undeterminable amount' [4]. Investigation of this principle has proceeded by examining the distribution of one observable both before and after the measurement of another observable, and attempting to relate the disturbance of the distribution to the accuracy of the measurement $[5,6,7]$. However, recent work by Ozawa $[8,9]$ shows that the momentum disturbance $\eta(P)$ due to a position measurement having inaccuracy $\epsilon(X)$ can in fact satisfy $\epsilon(X) \eta(P)=0$. Hence this principle needs to be formulated more carefully, presumably in relation to valid uncertainty relations such as $[8,9]$

$$
\epsilon(X) \eta(P)+\epsilon(X) \Delta P+\Delta X \eta(P) \geq \hbar / 2
$$

Finally, the fourth uncertainty principle above arises from Bohr's notion of complementarity [10], and restricts the degree to which joint information about observables can be obtained from a single experimental setup. However, previous formulations of corresponding uncertainty relations have only been given for special cases $[6,11,12,13,14,15,16,17]$. The most general of these are the Arthurs-Kelly type $[6,11,13,17]$, restricted to "universally unbiased" joint measurements; and the Martens-deMuynck type [14, 16], restricted to "non-ideal" joint measurements. For example, if a measurement apparatus simultaneously outputs two values $X_{J}$ and $P_{J}$, that are on average equal to the averages of $X$ and $P$ (for all input states), then $[6,11,13]$

$$
\Delta X_{J} \Delta P_{J} \geq \hbar
$$

The need to find general uncertainty relations quantifying complementarity, not subject to any restrictions on measurement, forms the subject of this paper.

To proceed, one first clearly needs to generalise what is meant by a "joint measurement". For example, neither "universally unbiased" or "non-ideal" joint measurements include experiments that are adapted in some way to particular subclasses of states. Yet Bohr defended complementarity against a number of thought experiments of this type [10], including the famous Einstein-Podolsky-Rosen (EPR) paradox [10, 18, 19]. In the latter case a joint measurement of $X$ and $P$ arises via simultaneous measurement of $X$ and $P^{\prime}$, where $P^{\prime}$ refers to the momentum of a (correlated) auxilary system. Such a joint measurement does not satisfy either of the "universal unbiasedness" or "non-ideal" restrictions mentioned above.

Indeed, in trying to place fundamental limits on the information which can simultaneously be gained about two complementary observables, one must consider any and all experimental setups, without restriction. The simplest and most general possible approach will therefore 
be taken in this paper: any measurement is considered to provide a joint measurement of any two observables. The corresponding logic is that (i) the result of a given measurement provides information; (ii) this information can be used to make an estimate of any given observable; and (iii) one may look for universal uncertainty relations associated with such estimates.

This approach solves the problem of what constitutes a joint measurement in a very general way (all measurements are permitted). However, there are still two possible strategies that may be followed to obtain general joint-measurement uncertainty relations. The first of these is simply to seek uncertainty relations which hold for any estimates, good or bad, of the observables. The second strategy is to throw away all the bad estimates, and only seek uncertainty relations for estimates that make the best possible use of any prior information (after all, why make a particular estimate if the information is available to make a better one?). Both strategies will be followed in this paper, and corresponding joint-measurement uncertainty relations are given in Secs. III and IV.

Note that the strategy of making the best use of any available prior information is of some interest in its own right, quite aside from joint measurements. For example, a measurement of position does not by itself provide a very useful basis for estimating energy. However, combining the measurement result with any information available about the system before measurement (eg, its average momentum, or its quantum state, or its entanglement with an auxilary system) can lead to a significantly improved estimate. More generally, prior information helps the experimenter place the detector to minimise null outcomes, and the quantum communications engineer to optimise the receiver. As emphasised by Trifonov et al. [17], even the "universally unbiased" bound in Eq. (4) is achieved only by choosing the experimental setup in dependence on prior information about the system to be measured (the "balance" parameter $b=\hbar \Delta X / \Delta P$ in [11], and the full polarisation state in [17]).

In Sec. II a general formula is given for the best possible estimate of an observable, based on an arbitrary measurement and prior information about the state of the system. This formula is related to and generalises expressions for "local expectation values" [20, 21, 22] and "weak values" $[23,24]$ of quantum observables. The best possible estimate is also determined for the case in which there is no prior information available. Examples are given for general energy estimation, and for estimation of the quadratures of a single-mode field using optical heterodyne detection. In the latter case the best possible estimates are related 
to the gradient of the Husimi Q-function.

In Sec. III a geometric uncertainty relation is given for the optimal estimates of any two observables from arbitrary measurement data, assuming that the state prior to measurement is known. This uncertainty relation implies a trade-off between the dispersions of the estimates (i.e., the spreads of the corresponding distributions), and the inaccuracies of the estimates (i.e., the degree to which the estimates successfully mimic the corresponding observables). A universal lower bound for the inaccuracy of any (possibly non-optimal) estimate is also given. For the case of heterodyne detection two further inequalities are derived, applying to the dispersions and to the inaccuracies of the estimates respectively. It is also shown that the optimal estimates resulting from a canonical joint measurement of position and momentum, on a known state, satisfy an uncertainty relation with a lower bound 1/4 of that in Eq. (4).

In Sec. IV a universal joint-measurement uncertainty relation is derived, valid for any estimates (optimal or otherwise) of two observables from a given experimental setup. The derivation shares a formal link with Ozawa's proof of Eq. (3) [8, 9], and modification of the derivation leads to stronger uncertainty relations such as Eq. (4) for the special case of universally unbiased measurements. Results are applied to a discussion of the abovementioned EPR paradox [18], and to quadrature estimation based on prior information about the averages of certain observables.

Some conclusions are given in Sec. V.

\section{MAKING THE BEST POSSIBLE ESTIMATE}

Consider an arbitrary measurement $\mathcal{M}$ having possible results $\{m\}$, and with statistics given by

$$
p(m \mid \rho)=\operatorname{tr}\left[\rho M_{m}\right]
$$

for a system described by density operator $\rho$. Since the probabilities must be positive and sum to unity, the operators $\left\{M_{m}\right\}$ must be positive and sum to the unit operator, and hence form a probability operator measure (POM) [1, 2, 25]. In the interests of generality, no further restrictions or specific measurement models are assumed.

A notation is adopted whereby a measurement, its corresponding POM, and the corresponding observable quantity, are all denoted by the same scripted character, eg, $\mathcal{M}$. Any 
Hermitian operators associated with the measurement will be denoted via related upper-case Roman characters, eg, $M_{m}$.

In some cases $\mathcal{M}$ may be equivalently described by a Hermitian operator $M$. In such a case $M_{m}$ is just the projection onto the eigenspace associated with eigenvalue $m$ of $M$, and $M=\sum_{m} m M_{m}$. If $M$ is non-degenerate with eigenkets $\{|m\rangle\}$, and the system is described by a pure state $\rho=|\psi\rangle\langle\psi|$, Eq. (5) reduces to the familiar expression $p(m \mid \psi)=|\langle m \mid \psi\rangle|^{2}$. As is well known, however, there are many non-trivial measurements that are not equivalent to some Hermitian operator acting on the Hilbert space of the system $[1,2,25]$.

As discussed in the Introduction, it may often be desirable to make an estimate of some observable based on the result of a given measurement and any available prior information. This Section is therefore concerned with answering the following question: for a quantum system described by density operator $\rho$, what is the best possible estimate one can make of some observable, $\mathcal{A}$, from a measurement of $\mathcal{M}$ with result $m$ ?

\section{A. Using prior information: a special case}

It is convenient, for the purpose of introducing the necessary concepts, to first consider the above question in the special case that $\mathcal{M}$ and $\mathcal{A}$ correspond to respective Hermitian operators $M$ and $A$. This case was also considered briefly in Ref. [26].

In particular, suppose that one seeks the best possible estimate of $A$ from the measurement of a Hermitian operator $M$ having eigenkets $\{|m\rangle\}$, where for simplicity it will be assumed that the system is in a known pure state $|\psi\rangle$ prior to measurement. It follows that any estimate $f(m)$ of $A$ from measurement result $M=m$ is equivalent to measurement of the Hermitian operator $f(M)=\sum_{m} f(m)|m\rangle\langle m|$. One may therefore represent the estimate as

$$
f(M)=A+N_{f},
$$

i.e., as the sum of the operator to be estimated, $A$, and a "noise operator", $N_{f}[6,8,13]$.

Now, the best possible estimate will of course depend on the criterion of optimality used to define "best possible". One obvious criterion is that the noise should be "small" on average, i.e., the quantity

$$
\left\langle\psi\left|N_{f}^{2}\right| \psi\right\rangle=\left\langle\psi\left|[f(M)-A]^{2}\right| \psi\right\rangle=D_{\psi}(f(M), A)^{2}
$$


should be small. Here $D_{\psi}(A, B):=\left\langle(A-B)^{2}\right\rangle^{1 / 2}$ denotes the statistical deviation between Hermitian operators $A$ and $B$ (see also Appendix). The best possible estimate is therefore defined as corresponding to the choice of $f$ that minimises the statistical deviation between the observable and its estimate.

To determine this best possible estimate, note that Eq. (6) can be rewritten as

$$
\begin{aligned}
D_{\psi}(f(M), A)^{2}= & \left\langle A^{2}\right\rangle+\sum_{m}|\langle m \mid \psi\rangle|^{2} f(m)^{2}-\sum_{m} f(m)[\langle\psi \mid m\rangle\langle m|A| \psi\rangle+\text { c.c. }] \\
= & \left\langle A^{2}\right\rangle-\sum_{m}|\langle m \mid \psi\rangle|^{2}\left[\operatorname{Re} \frac{\langle m|A| \psi\rangle}{\langle m \mid \psi\rangle}\right]^{2} \\
& +\sum_{m}|\langle m \mid \psi\rangle|^{2}\left[f(m)-\operatorname{Re} \frac{\langle m|A| \psi\rangle}{\langle m \mid \psi\rangle}\right]^{2} .
\end{aligned}
$$

Only the last term depends on the estimate, and is nonnegative. Hence the minimum possible statistical deviation or "noise" corresponds to the choice [26]

$$
f(m)=\tilde{A}_{\mathrm{opt}}(m \mid \psi):=\operatorname{Re} \frac{\langle m|A| \psi\rangle}{\langle m \mid \psi\rangle} .
$$

A tilde is used to distinguish this quantity from an operator.

Thus, when statistical deviation is used as the criterion of optimality, the optimal estimate of $A$, from measurement result $M=m$ on state $|\psi\rangle$, is given by $\tilde{A}_{\text {opt }}(m \mid \psi)$ in Eq. (8). It is only possible to make this estimate when the appropriate prior information - the state prior to measurement - is known. The case where no prior information is available is considered in Sec. II.C below.

The formula on the righthand side of Eq. (8) has in fact appeared previously in the literature in a variety of other contexts: as the "local expectation value" of the operator $A$ relative to $M$ for state $|\psi\rangle[20,21,22]$; as the "weak value" of the operator $A$ relative to pre-selected state $|\psi\rangle$ and post-selected state $|m\rangle$ [23, 24, 27, 28]; and as the "classical component" of $A$ with respect to $M$ for state $|\psi\rangle[26,29,30]$. However, only the above "estimation" context appears to provide a robust interpretation.

For example, the expression in Eq. (8) can be negative for a positive operator $A$, which undermines its interpretation as either a "value" or a "classical" component of $A$. In contrast, the fact that the best possible estimate of some positive observable, from the measurement of a second incompatible observable, can be negative on occasion merely provides a nice signature of the difference between quantum and classical estimation theory [31] (at least in the case where statistical deviation is used as the sole criterion of optimality). While one 
could of course restrict attention to estimates that fall within the eigenvalue range of $A$, the estimate in Eq. (8) still remains of fundamental interest in providing an absolute lower bound for the statistical deviation of any estimate. It should be noted that, in any case, all examples considered in this paper satisfy this restriction (with the exception of Eq. (16) in Sec. II.D).

\section{B. Using prior information: the general case}

The question posed at the beginning of this section, of how to determine the best possible estimate of an observable $\mathcal{A}$ from measurement of a general POM observable $\mathcal{M}$ on a system described by a known density operator $\rho$, may now be addressed. Clearly, it is first necessary to suitably generalise in some way the criterion of optimality used in the previous section.

For the case where $\mathcal{A}$ corresponds to some Hermitian operator $A$, the generalisation of statistical deviation turns out to be quite straightforward. In particular, as discussed in the Appendix, the natural definition of the statistical deviation between a Hermitian operator $A$ and a POM observable $\mathcal{B} \equiv\left\{B_{b}\right\}$, for a given state $\rho$, is

$$
D_{\rho}(A, \mathcal{B})^{2}:=\sum_{b} \operatorname{tr}\left[(A-b) \rho(A-b) B_{b}\right] .
$$

Note that this reduces to $D_{\psi}(A, B)$ in Eq. (6) when $\mathcal{B}$ corresponds to some Hermitian operator $B$. As shown in the Appendix, the derivation in Eq. (7) is easily generalised to give the optimal estimate

$$
f(m)=\tilde{A}_{\mathrm{opt}}(m \mid \rho):=\frac{\operatorname{tr}\left[\rho\left(M_{m} A+A M_{m}\right)\right]}{2 \operatorname{tr}\left[\rho M_{m}\right]}
$$

of $A$ from measurement result $\mathcal{M}=m$. The case where $\mathcal{A}$ does not correspond to a Hermitian operator is also discussed in the Appendix.

Eq. (10) clearly generalises Eq. (8), and has several properties worth noting. First, it follows via Eq. (5) that the optimal estimate is always unbiased, i.e.,

$$
\sum_{m} p(m \mid \rho) \tilde{A}_{\mathrm{opt}}(m \mid \rho)=\operatorname{tr}[\rho A]=\langle A\rangle .
$$

Second, if the system is initially in some eigenstate $|a\rangle$ of $A$, then $\tilde{A}_{\text {opt }}(m \mid \rho) \equiv a$, independently of the actual measurement result. Third, if $\mathcal{M}$ corresponds to an ideal measurement

of a Hermitian operator which commutes with $A$, one has the classical repeatability property

$$
\tilde{A}_{\text {opt }}(m \mid \bar{\rho})=\tilde{A}_{\text {opt }}(m \mid \rho)
$$


where $\bar{\rho}:=\sum_{m^{\prime}} M_{m^{\prime}} \rho M_{m^{\prime}}$ describes the post-measurement ensemble.

It is convenient to denote the physical observable associated with the optimal estimate in Eq. (10) by $\mathcal{A}_{\text {opt }}$. Measurement of $\mathcal{A}_{\mathrm{opt}}$ is carried out by measuring $\mathcal{M}$, and for result $m$ attributing the outcome $\tilde{A}_{\mathrm{opt}}(m \mid \rho)$ to $\mathcal{A}_{\mathrm{opt}}$. One may refer to $\mathcal{A}_{\mathrm{opt}}$ as the compatible component of $A$ with respect to $\mathcal{M}$. Note from Eq. (10) that compatible components form a linear algebra, i.e.,

$$
(\mathcal{A}+\lambda \mathcal{B})_{\mathrm{opt}}=\mathcal{A}_{\mathrm{opt}}+\lambda \mathcal{B}_{\mathrm{opt}}
$$

\section{No prior information}

Consider now the case where there is no information available about the state of the system prior to measurement. The statistical deviation therefore cannot be calculated, nor the estimates in Eqs. (8) and (10). The best possible estimate of $A$ from some measurement $\mathcal{M}$ must instead be defined via some state-independent criterion of optimality.

One suitable criterion is provided by a generalisation of the Hilbert-Schmidt distance $d(A, B)^{2}=\operatorname{tr}\left[(A-B)^{2}\right]$ between two Hermitian operators. Such a generalisation, $d(A, \mathcal{M})$, for a Hermitian operator $A$ and a POM observable $\mathcal{M}$, has recently been given [32]. The estimate "closest" to $A$, in the sense of minimising this distance, follows directly as (see Appendix)

$$
\tilde{A}_{\mathrm{opt}}^{0}(m):=\operatorname{tr}\left[A M_{m}\right] / \operatorname{tr}\left[M_{m}\right] .
$$

The physical observable corresponding to this estimate will be denoted by $\mathcal{A}_{\mathrm{opt}}^{0}$. Note from Eq. (10) that $\mathcal{A}_{\mathrm{opt}}^{0} \equiv \mathcal{A}_{\mathrm{opt}}$ in the case that $\rho$ is a maximally mixed state, i.e., $\rho \sim \mathbf{1}$.

The estimate in Eq. (13) is typically biased - after all, there is no prior information available about $\langle A\rangle$ to feed into such an estimate. However, depending on the relationship between $A$ and $\mathcal{M}$, it is possible for the estimate to be universally unbiased, as will be seen for heterodyne detection in Sec. II.D below. Further, in cases where the estimate is only linearly biased, it is possible to trade "distance" for "bias". For example, if

$$
\sum_{m} M_{m} \operatorname{tr}\left[A M_{m}\right] / \operatorname{tr}\left[M_{m}\right]=A+r
$$

for some constant $r$, then a universally unbiased estimate is obtained by replacing $A_{\mathrm{opt}}^{0}(m)$ with $A_{\mathrm{opt}}^{0}(m)-r$. 
As a more general example, consider the estimate of the spin, $\mathbf{S}=\hbar \sigma / 2$, of a spin- $1 / 2$ particle, from a measurement result $\mathbf{m}$ corresponding to a general POM $\left\{M_{\mathbf{m}}=q_{\mathbf{m}}(1+\right.$ $\sigma . \mathbf{m})\}$. Here $\mathbf{m}$ ranges over some subset $R$ of the Bloch ball, and $\left\{q_{\mathbf{m}}\right\}$ is any probability distribution on $R$ satisfying $\sum_{\mathbf{m}} q_{\mathbf{m}} \mathbf{m}=0$. The best possible estimate of $\mathbf{S}$ from result m follows from Eq. (13) as the linearly biased estimate $\hbar \mathbf{m} / 2$. The associated universally unbiased estimate is $\hbar \Lambda^{-1} \mathbf{m} / 2$, where $\Lambda$ denotes the matrix $\sum_{\mathbf{m}} q_{\mathbf{m}} \mathbf{m} \mathbf{m}^{T}$ (note that the inverse exists providing $R$ contains three linearly independent members). A similar result holds on general Hilbert spaces for trace-class $M_{m}$ and $A$, with the components of $\sigma$ replaced by a linearly independent basis set of trace-free Hermitian operators.

\section{Example: energy estimation}

Making the best possible estimate of energy from the measurement of various observables is considered here, to indicate the types of expressions that can arise.

First, for a particle with Hamiltonian operator $H$, consider the case where all that is known about the system is that it is in thermal equilibrium corresponding to temperature $T$. The particle is therefore described by the density operator proportional to $e^{-\beta H}$, where $\beta=1 /(k T)$. It follows from Eq. (10) that the optimal estimate of the energy of the system, from measurement result $\mathcal{M}=m$, is given by

$$
\tilde{E}_{\text {opt }}(m \mid T)=-(\partial / \partial \beta) \ln \operatorname{tr}\left[e^{-\beta H} M_{m}\right]
$$

Thus $\operatorname{tr}\left[e^{-\beta H} M_{m}\right]$ is a kind of generalised partition function. For the particular example of a position measurement, on a 1-dimensional harmonic oscillator of mass $m$ and frequency $\omega$, one obtains the quadratic estimate

$$
\tilde{E}_{\text {opt }}(x \mid T)=A_{T}+B_{T} x^{2}
$$

where $A_{T}=(1 / 2) \hbar \omega \operatorname{coth}(\beta \hbar \omega)$ and $B_{T}=(1 / 2) m \omega^{2} \operatorname{sech}^{2}(\beta \hbar \omega / 2)$. Note that in the zerotemperature limit this estimate reduces to the groundstate energy $\hbar \omega / 2$, independently of the actual measurement result $x$. In the classical limit $\hbar \rightarrow 0$ the estimate reduces to $(1 / 2) k T+(1 / 2) m \omega^{2} x^{2}$, i.e., to the sum of the average kinetic energy and the potential energy (this result holds more generally).

Second, for a particle with Hamiltonian $H=P^{2} /(2 m)+V(X)$ in a known pure state $|\psi\rangle$, the best possible estimate of energy from a measurement of position $X$ follows from Eq. (8) 
as

$$
\tilde{E}_{\text {opt }}(x \mid \psi)=|\nabla S|^{2} /(2 m)+V(x)+Q(x),
$$

where $\langle x \mid \psi\rangle=R \exp (i S / \hbar)$, and $Q(x)=-\hbar^{2} /(2 m) \nabla^{2} R / R$ is the so-called "quantum potential" [21]. Note that $Q(x)$ arises here in the context of the best possible estimate of the kinetic energy [i.e., the possibly negative quantity $|\nabla S|^{2} /(2 m)+Q(x)$ ], with no relation to a real potential energy.

Third and finally, consider a single-mode optical field with annihilation operator $a$ and Hamiltonian $H=\hbar \omega a^{\dagger} a$. An inefficient measurement of photon number, via a photodetector having quantum efficiency $\eta$, corresponds to the POM $\left\{M_{m}(\eta)\right\}$ with number-state expansion $M_{m}(\eta)=\sum_{r}|m+r\rangle\langle m+r|{ }^{m+r} C_{r} \eta^{m}(1-\eta)^{r}[16,25]$. If there is no prior information about the state of the field prior to measurement, the best possible estimate of the energy of the field then follows from Eq. (13) as

$$
\tilde{E}_{\mathrm{opt}}^{0}(m)=\hbar \omega[(m+1) / \eta-1]
$$

using the identity $\sum_{r}{ }^{m+r} C_{r} x^{r}=(1-x)^{-m-1}$. This estimate is linearly biased [with $r=$ $1 / \eta-1$ in Eq. (14)], with the associated univerally unbiased estimate given by $\hbar \omega m / \eta$.

\section{E. Example: heterodyne detection}

For a single-mode optical field with annihilation operator $a$, the quadrature observables $X_{1}=\left(a+a^{\dagger}\right) / 2, X_{2}=\left(a-a^{\dagger}\right) / 2 i$ have commutator $\left[X_{1}, X_{2}\right]=i / 2$, and hence are analogous to the position and momentum observables of a quantum particle (with $\hbar$ replaced by $1 / 2$ ). In particular, $X_{1}$ and $X_{2}$ cannot be measured simultaneously to an arbitrary accuracy.

However, in optical heterodyne detection, one introduces an auxilary imageband field with annihilation operator $b$, and simultaneously measures the real and imaginary parts of the operator $a+b^{\dagger}[33,34,35]$, i.e., one measures the commuting observables

$$
X_{1, J}=X_{1}+Y_{1}, \quad X_{2, J}=X_{2}-Y_{2},
$$

where $Y_{1}$ and $Y_{2}$ denote the corresponding quadratures of the imageband field. This may be interpreted as corresponding to an approximate joint measurement of $X_{1}$ and $X_{2}$, subject to imageband noise. 
Clearly this joint measurement is formally equivalent to the canonical joint measurement of position $X$ and momentum $P$ of a quantum particle, referred to in the Abstract, where one introduces an auxilary particle with corresponding observables $X^{\prime}$ and $P^{\prime}$, and simultaneously measures

$$
X_{J}=X+X^{\prime}, \quad P_{J}=P-P^{\prime} .
$$

This formal equivalence allows one to map results from one context to the other.

For the general case of an uncorrelated imageband field described by density operator $\rho_{i}$, the measurement statistics of heterodyne detection correspond to a continuous POM $\left\{M_{\alpha}\right\}$, with $M_{\alpha}=\pi^{-1} D(\alpha) \rho_{i}^{\prime} D(\alpha)^{\dagger}[36]$. Here $\alpha$ denotes the complex eigenvalue $\alpha_{1}+i \alpha_{2}$ of $a+b^{\dagger}$, $D(\alpha)$ denotes the Glauber displacement operator $\exp \left(\alpha a^{\dagger}-\alpha^{*} a\right)$, and $\rho_{i}^{\prime}$ is defined by

$$
\rho_{i}^{\prime}:=\sum_{m, n}|m\rangle_{a}\langle n|(-1)^{m+n}\left\langle m\left|\rho_{i}\right| n\right\rangle_{b}^{*},
$$

where the subscripts $a$ and $b$ refer to number states of the signal and imageband fields respectively.

For simplicity, attention will be restricted in what follows to the case of a vacuum-state imageband field. For this case $\rho_{i}^{\prime}=\rho_{i}=|0\rangle\langle 0|$, and hence the measurement is described by the well known coherent-state POM [1, 2, 25, 35], with

$$
M_{\alpha}=\pi^{-1}|\alpha\rangle\langle\alpha|,
$$

and associated measurement statistics given by the Husimi Q-function

$$
Q(\alpha)=\pi^{-1}\langle\alpha|\rho| \alpha\rangle
$$

Now, suppose first that there is no prior information available about the state of the field. The best possible estimate of the quadrature $X_{1}$, from measurement result $\alpha$, then follows from Eq. (13) as the estimate

$$
\tilde{X}_{1, \mathrm{opt}}^{0}(\alpha)=\left\langle\alpha\left|X_{1}\right| \alpha\right\rangle /\langle\alpha \mid \alpha\rangle=\alpha_{1}
$$

Similarly, the best possible estimate of $X_{2}$ in the case of no prior information is given by

$$
\tilde{X}_{2, \text { opt }}^{0}(\alpha)=\alpha_{2}
$$

Thus the best possible estimates are directly given by the measurement result $\alpha$, i.e., they are equivalent to measurement of $X_{1, J}$ and $X_{2, J}$ in Eq. (18). More generally, the best possible 
estimate of a general Hermitian observable $f\left(a, a^{\dagger}\right)$, when no prior information is available, follows from Eq. (13) as $f^{(n)}\left(\alpha, \alpha^{*}\right)$, where $f^{(n)}$ denotes the normally-ordered form of $f$.

The situation changes markedly when prior information about the state of the system $i s$ available. In particular, the best possible estimate of $X_{1}$ for a measurement on a known state $\rho$ follows via Eq. (10) as

$$
\begin{aligned}
\tilde{X}_{1, \mathrm{opt}}(\alpha \mid \rho) & =\left\langle\alpha\left|X_{1} \rho+\rho X_{1}\right| \alpha\right\rangle /\langle\alpha|\rho| \alpha\rangle / 2 \\
& =\frac{1}{2} \operatorname{Re}\{\alpha+\langle\alpha|a \rho| \alpha\rangle /\langle\alpha|\rho| \alpha\rangle\}
\end{aligned}
$$

Thus the direct "no prior information" estimate, $\alpha_{1}=\operatorname{Re} \alpha$ in Eq. (21), provides only half of the input to the more general estimate of $X_{1}$. The other half depends on the state, and is typically a highly nonlinear function of both $\alpha_{1}$ and $\alpha_{2}$. One has a similar estimate

$$
\tilde{X}_{2, \text { opt }}(\alpha \mid \rho)=\frac{1}{2} \operatorname{Im}\{\alpha+\langle\alpha|a \rho| \alpha\rangle /\langle\alpha|\rho| \alpha\rangle\}
$$

for the quadrature $X_{2}$, where again the "no prior information" estimate, $\alpha_{2}=\operatorname{Im} \alpha$, provides only half the input.

Further insight into these best possible estimates is gained by expressing them solely in terms of the Husimi Q-function $Q(\alpha)$ in Eq. (20). In particular, noting that variation with respect to $\alpha$ gives

$$
\begin{aligned}
\delta\langle\alpha|\rho| \alpha\rangle & =\left\langle\alpha\left|D(\delta \alpha)^{\dagger} \rho D(\delta \alpha)\right| \alpha\right\rangle-\langle\alpha|\rho| \alpha\rangle \\
& =\left\langle\alpha\left|\left[\rho, a^{\dagger}\right]\right| \alpha\right\rangle \delta \alpha+\langle\alpha|[a, \rho]| \alpha\rangle \delta \alpha^{*}
\end{aligned}
$$

one may replace $a \rho$ by $[a, \rho]+\rho a$ in Eqs. (23) and (24) to obtain

$$
\tilde{X}_{j, \mathrm{opt}}(\alpha \mid \rho)=\alpha_{j}+(1 / 4)\left(\partial / \partial \alpha_{j}\right) \log Q
$$

for $j=1,2$. Hence the best possible estimates differ significantly from $\alpha_{1}$ and $\alpha_{2}$ precisely when the gradient of the logarithm of the probability distribution, at the point corresponding to the measurement outcome, is large.

As examples, consider the cases where the field is known to be in a coherent state $|\beta\rangle$, and in a number state $|n\rangle$. One then finds from Eqs. (23) and (24), or equivalently from Eq. (26),

$$
\begin{aligned}
\tilde{X}_{1, \mathrm{opt}}(\alpha \mid \beta)+i \tilde{X}_{2, \mathrm{opt}}(\alpha \mid \beta) & =\frac{1}{2}(\alpha+\beta), \\
\tilde{X}_{1, \mathrm{opt}}(\alpha \mid n)+i \tilde{X}_{2, \mathrm{opt}}(\alpha \mid n) & =\frac{1}{2} \alpha\left(1+n /|\alpha|^{2}\right)
\end{aligned}
$$


respectively.

Finally, to preview the effect of prior information on joint-measurement uncertainty relations, the uncertainties of the estimates $\mathcal{X}_{1 \text {,opt }}$ and $\mathcal{X}_{2 \text {,opt }}$ will be calculated here for the above coherent-state example. These estimates are equivalent to the measurement of $\left(X_{1, J}+\beta\right) / 2$ and $\left(X_{2, J}+\beta\right) / 2$ respectively, and hence, using Eq. (18),

$$
\operatorname{Var} \mathcal{X}_{1, \mathrm{opt}}=(1 / 4) \operatorname{Var} X_{1, J}=\left(\operatorname{Var} X_{1}+\operatorname{Var} Y_{1}\right) / 4=1 / 8
$$

with a similar result for $\operatorname{Var} \mathcal{X}_{2, \text { opt }}$. One therefore obtains the uncertainty product

$$
\Delta \mathcal{X}_{1, \mathrm{opt}} \Delta \mathcal{X}_{2, \mathrm{opt}}=1 / 8
$$

for this example, which is four times better than the corresponding product,

$$
\Delta X_{1, J} \Delta X_{2, J}=1 / 2
$$

for the case when no prior information about the state is available. It will be shown in the following section that this factor of 4 improvement is the ultimate limit.

\section{UNCERTAINTY RELATIONS FOR OPTIMAL ESTIMATES}

\section{A. Dispersion vs inaccuracy: a geometric uncertainty relation}

There are two types of contribution to the "uncertainty" of an estimate. The first, dispersion, is related to the statistics of the estimate itself, whereas the second, inaccuracy, is related to how well the estimate does its job of estimating a given observable. These two types of uncertainty are to some degree complementary, and it will be seen that for optimal estimates they are linked by a very simple uncertainty relation.

To characterise dispersion, let $\mathcal{A}_{f}$ denote the observable corresponding to a general estimate of $A$ from measurement $\mathcal{M}$, where outcome $m$ of $\mathcal{M}$ corresponds to outcome $f(m)$ of $\mathcal{A}_{f}$. The statistics of the estimate are completely determined by the statistics of $\mathcal{M}$ and the choice of $f$, and in particular the root mean square deviation of $\mathcal{A}_{f}$ may be calculated in the usual way as

$$
\left(\Delta \mathcal{A}_{f}\right)^{2}=\sum_{m} p(m \mid \rho) f(m)^{2}-\left[\sum_{m} p(m \mid \rho) f(m)\right]^{2},
$$


where the outcome probability $p(m \mid \rho)$ is given by Eq. (5). This quantity will be used as a measure of the dispersion of the estimate.

To characterise the inaccuracy of the estimate $\mathcal{A}_{f}$, one requires a measure $\epsilon\left(\mathcal{A}_{f}\right)$ of the degree to which the estimate differs from the observable being estimated. In particular, it should be nonnegative, and vanish in the case of a perfect estimate (i.e., $\mathcal{A}_{f} \equiv \mathcal{A}$ ). The statistical deviation used in Eq. (9) satisfies these properties, and hence the quantity

$$
\epsilon\left(\mathcal{A}_{f}\right):=D_{\rho}\left(A, \mathcal{A}_{f}\right)
$$

will be used as a measure of inaccuracy of the estimate. Note from Eq. (6) that, for Hermitian observables, this measure is just the mean deviation of the noise operator associated with the estimate $[6,8,13]$. Note also that the optimal estimates of Sec. II based on prior information about the state of the system are precisely those estimates having the minimum possible inaccuracy: $\epsilon\left(\mathcal{A}_{f}\right) \geq \epsilon\left(\mathcal{A}_{\text {opt }}\right)$.

It follows immediately from Eq. (A3) of the Appendix that

$$
(\Delta A)^{2}=\left(\Delta \mathcal{A}_{\mathrm{opt}}\right)^{2}+\epsilon\left(\mathcal{A}_{\mathrm{opt}}\right)^{2}
$$

i.e., the dispersion and inaccuracy of the best possible estimate form the sides of a rightangled triangle having hypotenuse $\Delta A$. Thus there is a fundamental tradeoff between dispersion and inaccuracy, valid for any measurement $\mathcal{M}$. This tradeoff may be geometrically represented by the constraint that $\mathcal{A}_{\text {opt }}$ lies on a circle (or hypersphere) having diametrically opposed "poles" $A$ and $\langle A\rangle$. These poles correspond to the optimal estimates for $\mathcal{M} \equiv \mathcal{A}$ (i.e., a perfect estimate) and $\mathcal{M} \equiv \mathbf{1}$ (i.e., a trivial estimate) respectively. Alternatively, one may represent the tradeoff by a circle of radius $\Delta A$ in the dispersion-inaccuracy plane, with zero inaccuracy and zero dispersion corresponding to the cases $\mathcal{M} \equiv \mathcal{A}$ and $\mathcal{M} \equiv \mathbf{1}$ respectively.

The above geometric property, and the standard uncertainty relation $\triangle A \Delta B \geq$ $|\langle[A, B]\rangle| / 2$, allows one to immediately write down a general uncertainty relation for the best possible estimates of two Hermitian operators $A$ and $B$ from an arbitrary POM measurement $\mathcal{M}$ :

$$
\left[\left(\Delta \mathcal{A}_{\mathrm{opt}}\right)^{2}+\epsilon\left(\mathcal{A}_{\mathrm{opt}}\right)^{2}\right]^{1 / 2}\left[\left(\Delta \mathcal{B}_{\mathrm{opt}}\right)^{2}+\epsilon\left(\mathcal{B}_{\mathrm{opt}}\right)^{2}\right]^{1 / 2} \geq|\langle[A, B]\rangle| / 2
$$

Thus, for a non-zero lower bound, one cannot make both estimates arbitrarily accurate while making the corresponding dispersions arbitrarily small, no matter what measurement scheme 
is adopted. Note that the lower bound is achieved if and only if the system is in a minimumuncertainty state of $A$ and $B$.

\section{B. Incompatibility implies inaccuracy}

One has the useful lower bound

$$
\epsilon\left(\mathcal{A}_{\text {opt }}\right)^{2} \geq \sum_{m} \frac{\left|\operatorname{tr}\left[\rho\left(A M_{m}-M_{m} A\right)\right]\right|^{2}}{4 \operatorname{tr}\left[\rho M_{m}\right]}
$$

for the inaccuracy of the best possible estimate. Equality holds in the case that $\rho$ is pure and $\mathcal{M}$ is complete (i.e., with $M_{m}=|m\rangle\langle m|$ for all $m$ ), and hence in particular for the case of heterodyne detection with pure signal and imageband fields. Note that since the optimal estimate of $A$ has, by definition, the best possible accuracy, the righthand side of Eq. (32) in fact provides a lower bound for the inaccuracy of any estimate of $A$ from $\mathcal{M}$, and hence is universal.

The lower bound is non-trivial whenever $\left\langle\left[A, M_{m}\right]\right\rangle$ does not vanish for some $m$, i.e., whenever $A$ and $\mathcal{M}$ are incompatible for state $\rho$. Hence, one can never make a perfect estimate of one observable from the measurement of a second incompatible observable. When $\mathcal{A}$ and $\mathcal{M}$ are a pair of canonically conjugate observables, the lower bound is proportional to the Fisher information of $\mathcal{M}$, and the case of equality corresponds to an "exact uncertainty relation" for $\mathcal{A}$ and $\mathcal{M}[26,29]$.

Eq. (32) generalises Eq. (47) of Ref. [26] (in the context of exact uncertainty relations) and Eq. (14) in Ref. [28] (in the context of weak values), to general POM measurements $\mathcal{M}$. It follows via the Schwarz inequality $\left|\operatorname{tr}\left[K^{\dagger} L\right]\right|^{2} \leq \operatorname{tr}\left[K^{\dagger} K\right] \operatorname{tr}\left[L^{\dagger} L\right]$, which gives

$$
\left|\operatorname{tr}\left[\rho A M_{m}\right]\right|^{2} \leq \operatorname{tr}\left[\rho M_{m}\right] \operatorname{tr}\left[\rho A M_{m} A\right]
$$

for the choice $K=\rho^{1 / 2} M_{m}^{1 / 2}, L=\rho^{1 / 2} A M_{m}^{1 / 2}$. Noting the first equality in Eq. (A3) of the Appendix, and using $\left(z+z^{*}\right)^{2}=4|z|^{2}-\left|z-z^{*}\right|^{2}$ for $z=\operatorname{tr}\left[\rho A M_{m}\right]$ appearing in the optimal estimate in Eq. (10), then leads directly to Eq. (32). Equality holds for $K$ proportional to $L$, and hence in particular for a complete measurement on a pure state. 


\section{Example: heterodyne detection}

For heterodyne detection with a vacuum-state imageband field, as discussed in Sec. II.E, it will be shown that one has the further independent inequalities

$$
\begin{aligned}
\Delta \mathcal{X}_{1, \mathrm{opt}} \Delta \mathcal{X}_{2, \mathrm{opt}} & \geq 1 / 8 \\
\epsilon\left(\mathcal{X}_{1, \mathrm{opt}}\right)^{2}+\epsilon\left(\mathcal{X}_{2, \mathrm{opt}}\right)^{2} & \geq 1 / 4
\end{aligned}
$$

for the dispersions and the inaccuracies of the best possible estimates. The first relation is saturated for coherent states, and the second relation is saturated for all pure states.

Note that for the analogous case of a canonical joint measurement of position and momentum as discussed in Sec. II.D (with the auxilary system in a minimum uncertainty state), it follows immediately from Eq. (33) that one has the corresponding uncertainty relation

$$
\Delta \mathcal{X}_{\mathrm{opt}} \Delta \mathcal{P}_{\mathrm{opt}} \geq \hbar / 4
$$

improving on the "universally unbiased" lower bound in Eq. (4) by a factor of 4. Thus, even when one has complete information about the system prior to measurement, there is still a fundamental lower bound to the product of the dispersions of the optimal estimates.

To prove Eqs. (33) and (34), recall that the $2 \times 2$ covariance matrix $C$ for two random variables $A_{1}, A_{2}$ is given by $C_{j k}:=\left\langle A_{j} A_{k}\right\rangle-\left\langle A_{j}\right\rangle\left\langle A_{k}\right\rangle$. Hence the covariance matrix $C^{\mathrm{opt}}$ of the optimal quadrature estimates follows via Eqs. (21), (22) and (26) as

$$
\begin{aligned}
C_{j k}^{\mathrm{opt}} & =\left\langle\alpha_{j} \alpha_{k}\right\rangle+\frac{1}{4} \int d^{2} \alpha\left(\alpha_{j} \frac{\partial Q}{\partial \alpha_{k}}+\alpha_{k} \frac{\partial Q}{\partial \alpha_{j}}\right)+\frac{1}{16} F_{j k}^{Q} \\
& =C_{j k}^{Q}+(1 / 16) F_{j k}^{Q}-(1 / 2) \delta_{j k} .
\end{aligned}
$$

Here $C^{Q}$ is the covariance matrix for the joint-quadrature observables $X_{1, J}$ and $X_{2, J}$ in Eq. (18), $F^{Q}$ denotes the Fisher information matrix of the Husimi Q-function with [37]

$$
F_{j k}^{Q}:=\int d^{2} \alpha Q\left(\partial \log Q / \partial \alpha_{j}\right)\left(\partial \log Q / \partial \alpha_{k}\right),
$$

and integration by parts has been used to obtain the second line.

Now, if $F_{j}$ denotes the Fisher information of the marginal distribution $Q_{j}\left(\alpha_{j}\right)$ for $\alpha_{j}$, then the Cramer-Rao inequality from classical statistics [37] yields $F_{j} \geq 1 / C_{j j}^{Q}$. One also has

$$
0 \leq \int d^{2} \alpha Q(\alpha)\left[\left(\partial \log Q / \partial \alpha_{j}\right)-\left(\partial \log Q_{j} / \partial \alpha_{j}\right)\right]^{2}=F_{j j}^{Q}-F_{j}
$$


Substitution of these inequalities into Eq. (36) then yields

$$
C_{j j}^{\mathrm{opt}} \geq C_{j j}^{Q}+1 /\left(16 C_{j j}^{Q}\right)-1 / 2
$$

Writing $\operatorname{Var} X_{1}=\gamma r / 4, \operatorname{Var} X_{2}=\gamma /(4 r)$, with $\gamma \geq 1$ (to satisfy the standard uncertainty relation for the quadratures) and $r \geq 0$, and noting from Eq. (18) that $C_{j j}^{Q}=\operatorname{Var} X_{j}+1 / 4$, therefore leads to

$$
C_{11}^{\mathrm{opt}} C_{22}^{\mathrm{opt}} \geq \frac{\gamma^{3}}{16\left(\gamma^{2}-1\right)}\left[\frac{\gamma}{\gamma+r}-\frac{1}{\gamma r+1}\right] .
$$

Minimising the righthand side with respect to $r$ gives $r=1$; minimising the resulting expression with respect to $\gamma \geq 1$ then gives $\gamma=1$; and Eq. (33) immediately follows.

Finally, to obtain Eq. (34), note first that combining Eqs. (20), (25), (32) and (37) gives

$$
\epsilon\left(\mathcal{X}_{1, \mathrm{opt}}\right)^{2} \geq F_{22}^{Q} / 16, \quad \epsilon\left(\mathcal{X}_{2, \mathrm{opt}}\right)^{2} \geq F_{11}^{Q} / 16
$$

where equality holds for all pure states. Thus the accuracy of the estimate of one quadrature is related to the Fisher information of the other quadrature. Moreover, taking the trace of $C^{\text {opt }}$ in Eq. (36) and using the Euclidean relation in Eq. (30), one also has

$$
\epsilon\left(\mathcal{X}_{1, \text { opt }}\right)^{2}+\epsilon\left(\mathcal{X}_{2, \text { opt }}\right)^{2}=1 / 2-\left(F_{11}^{Q}+F_{22}^{Q}\right) / 16
$$

(giving an upper bound of half a photon for the lefthand side). Comparison with Eq. (38) immediately yields the known relation [37]

$$
F_{11}^{Q}+F_{22}^{Q} \leq 4
$$

for the trace of the Fisher information matrix, which when inserted back into the previous expression yields Eq. (34) as desired.

\section{UNIVERSAL JOINT-MEASUREMENT UNCERTAINTY RELATION}

\section{A. Arbitrary estimates}

The uncertainty relation to be derived in this section applies to any estimates of two Hermitian operators $A$ and $B$ from a general measurement $\mathcal{M}$. Unlike the geometric uncertainty relation of the previous section, it is valid for both optimal and non-optimal estimates, and is independent of whether or not any prior information about the system is available. The 
associated derivation may be modified to obtain the more restrictive uncertainty relations satisfied by universally unbiased estimates, such as Eq. (4).

Suppose then that $f(m)$ and $g(m)$ are general estimates for $A$ and $B$ respectively, for measurement result $\mathcal{M}=m$. These estimates thus correspond to two compatible observables $\mathcal{A}_{f}$ and $\mathcal{B}_{g}$, measured by measuring $\mathcal{M}$ and for outcome $m$ assigning the values $f(m)$ and $g(m)$ respectively. It will be shown that these estimates satisfy the universal uncertainty relation

$$
\Delta \mathcal{A}_{f} \epsilon\left(\mathcal{B}_{g}\right)+\epsilon\left(\mathcal{A}_{f}\right) \Delta \mathcal{B}_{g}+\epsilon\left(\mathcal{A}_{f}\right) \epsilon\left(\mathcal{B}_{g}\right) \geq|\langle[A, B]\rangle| / 2 .
$$

This uncertainty relation is therefore a fundamental expression of the limitations imposed by complementarity on quantum systems.

As a very simple example, suppose that one makes no physical measurement at all, but simply estimates $A=0$ and $B=0$ on every occasion. Then clearly the dispersions of the estimates vanish: $\Delta \mathcal{A}_{f}=\Delta \mathcal{B}_{g}=0$. The universal uncertainty relation Eq. (40) then implies that the product of the inaccuracies of such trivial estimates is non-trivially bounded below, i.e.,

$$
\epsilon\left(\mathcal{A}_{f}\right) \epsilon\left(\mathcal{B}_{g}\right) \geq|\langle[A, B]\rangle| / 2 .
$$

As a less trivial example, suppose that the position $X$ of a quantum particle is measured, and used to estimate both the position and the momentum of the particle. It is natural to choose $\mathcal{X}_{f} \equiv X$ (this is in fact the optimal estimate, whether or not any prior information is available). This estimate of $X$ is perfectly accurate, i.e., $\epsilon\left(\mathcal{X}_{\mathrm{f}}\right)=0$, and hence from Eq. (40)

$$
\Delta X \epsilon\left(\mathcal{P}_{g}\right) \geq \hbar / 2
$$

for any corresponding estimate $\mathcal{P}_{g}$ of the momentum. Note that this is a stronger result than the corresponding geometric uncertainty relation following from Eq. (31).

The proof of Eq. (40) proceeds via a formal trick - the representation of the measurement $\mathcal{M}$ as a Hermitian operator $M^{\prime}$ on an extended Hilbert space. This representation (a Naimark extension) preserves the statistical deviation between observables, while allowing one to exploit algebraic properties of Hermitian operators. Any such representation can be used for the proof, however, the choice of a product space representation is perhaps the simplest.

In particular, for a given $\mathrm{POM} \mathcal{M} \equiv\left\{M_{m}\right\}$ one can always (formally) introduce an auxilary system described by some fixed state $\rho^{\prime}$, and a Hermitian operator $M^{\prime}$ acting on 
the tensor product of the system and auxilary system Hilbert spaces, such that the statistics of $\mathcal{M}$ and $M^{\prime}$ are identical $[1,2,25]$, i.e.,

$$
p(m \mid \rho)=\operatorname{tr}\left[\rho M_{m}\right]=\operatorname{tr}\left[\rho \otimes \rho^{\prime} M_{m}^{\prime}\right]
$$

for all density operators $\rho$ and outcomes $m$, where $M_{m}^{\prime}$ denotes the projection on the eigenspace associated with eigenvalue $m$ of $M^{\prime}$. Note that this representation is used here as a formal mathematical device only, with no physical content.

It follows immediately from Eq. (41) that the statistics of general estimates $\mathcal{A}_{f}$ and $\mathcal{B}_{g}$ are equivalent to the statistics of the (commuting) Hermitian operators $f\left(M^{\prime}\right)$ and $g\left(M^{\prime}\right)$ respectively. Further, if $\left\{\left|s^{\prime}\right\rangle\right\}$ denotes a complete set of kets for the auxilary Hilbert space, Eq. (41) yields the partial trace relation

$$
\operatorname{tr}_{\rho^{\prime}}\left[\rho^{\prime} M_{m}^{\prime}\right]:=\sum_{s^{\prime}}\left\langle s^{\prime}\left|\rho^{\prime} M_{m}^{\prime}\right| s^{\prime}\right\rangle=M_{m}
$$

Hence, using Eqs. (A1) and (A2) of the Appendix, one has

$$
\begin{aligned}
D_{\rho \otimes \rho^{\prime}}\left(A, f\left(M^{\prime}\right)\right)^{2} & =\left\langle A^{2}\right\rangle+\left\langle f\left(M^{\prime}\right)^{2}\right\rangle-\sum_{m} f(m) \operatorname{tr}\left[\rho \otimes \rho^{\prime}\left(A M_{m}^{\prime}+M_{m}^{\prime} A\right)\right] \\
& =\left\langle A^{2}\right\rangle+\left\langle\mathcal{A}_{f}^{2}\right\rangle-\sum_{m} f(m)\left\{\operatorname{tr}_{\rho}\left[\rho A \operatorname{tr}_{\rho^{\prime}}\left[\rho^{\prime} M_{m}^{\prime}\right]\right]+c . c .\right\} \\
& =\left\langle A^{2}\right\rangle+\left\langle\mathcal{A}_{f}^{2}\right\rangle-\operatorname{tr}\left[\rho A \bar{A}_{f}+\bar{A}_{f} A \rho\right] \\
& =D_{\rho}\left(A, \mathcal{A}_{f}\right)^{2}=\epsilon\left(\mathcal{A}_{f}\right)^{2}
\end{aligned}
$$

and thus the representation preserves statistical deviation and inaccuracy. Writing $\delta A=$ $A-f\left(M^{\prime}\right), \delta B=B-g\left(M^{\prime}\right)$, it follows that $\epsilon\left(\mathcal{A}_{f}\right)^{2}=\left\langle(\delta A)^{2}\right\rangle$ and $\epsilon\left(\mathcal{B}_{g}\right)^{2}=\left\langle(\delta B)^{2}\right\rangle$, and hence that

$$
\begin{aligned}
|\langle[A, B]\rangle| & =\left|\left\langle\left[f\left(M^{\prime}\right)+\delta A, g\left(M^{\prime}\right)+\delta B\right]\right\rangle\right| \\
& \leq\left|\left\langle\left[f\left(M^{\prime}\right), \delta B\right]\right\rangle\right|+\left|\left\langle\left[\delta A, g\left(M^{\prime}\right)\right]\right\rangle\right|+|\langle[\delta A, \delta B]\rangle| \\
& \leq 2 \Delta f\left(M^{\prime}\right) \epsilon\left(\mathcal{B}_{g}\right)+2 \epsilon\left(\mathcal{A}_{f}\right) \Delta g\left(M^{\prime}\right)+2 \epsilon\left(\mathcal{A}_{f}\right) \epsilon\left(\mathcal{B}_{g}\right),
\end{aligned}
$$

using the triangle inequality and the Schwarz inequality $\left\langle(K-k)^{2}\right\rangle\left\langle L^{2}\right\rangle \geq|\langle[K, L]\rangle|^{2} / 4$ (in a manner formally similar to Ozawa's proof of Eq. (3) $[8,9])$. The last line is equivalent to the universal uncertainty relation in Eq. (40).

Finally, the above derivation may be modified to obtain a stronger uncertainty relation, valid for the special case of universally unbiased estimates of $A$ and $B[6,11,13]$. In 
particular, the requirements that $\left\langle\mathcal{A}_{f}\right\rangle=\langle A\rangle,\left\langle\mathcal{B}_{g}\right\rangle=\langle B\rangle$ for all states $\rho$ implies via Eq. (42) that

$$
A=\operatorname{tr}_{\rho^{\prime}}\left[\rho^{\prime} f\left(M^{\prime}\right)\right], \quad B=\operatorname{tr}_{\rho^{\prime}}\left[\rho^{\prime} g\left(M^{\prime}\right)\right]
$$

Hence $\left.\operatorname{tr}_{\rho^{\prime}}\left[\rho^{\prime} A g\left(M^{\prime}\right)\right]=A B=\operatorname{tr}_{\rho^{\prime}}\left[\rho^{\prime} f\left(M^{\prime}\right) B\right)\right]$, implying that $\langle[\delta A, \delta B]\rangle=\langle-[A, B]\rangle$. Thus, with no triangle inequality being necessary, the Schwarz inequality yields

$$
\epsilon\left(\mathcal{A}_{f}\right) \epsilon\left(\mathcal{B}_{g}\right) \geq|\langle[\delta A, \delta B]\rangle| / 2=|\langle[A, B]\rangle| / 2 \text {. }
$$

The joint uncertainty relation for universally unbiased joint measurements of position and momentum, Eq. (4), is a straightforward consequence of this result $[6,11,13]$.

\section{B. Example: EPR estimates}

The notion that the properties of position and motion are incompatible goes back nearly 2500 years to Zeno of Elea (who resolved the issue by concluding that motion was impossible). However, in classical physics this notion was rejected due to the existence of a consistent model: one can simultaneously define both the position and motion of a classical system by assuming that it follows a (differentiable) continuous trajectory in configuration space. Unfortunately, in the standard quantum formalism there are no such trajectories for physical systems, and a new resolution of the issue is needed.

In the standard interpretation of quantum mechanics, as formulated by Heisenberg and Bohr $[4,10]$, one takes the view that the properties of position and motion are indeed incompatible, in the sense of being unable to be accurately defined/measured simultaneously, and to this extent agrees with Zeno. However, others (most notably Einstein) have argued that the quantum formalism is in fact incomplete, and that quantum systems can in particular have simultaneously well-defined physical values of position and momentum [18]. It has since been shown that any such "hidden variable" interpretation requires the existence of a mutual influence or conspiracy between a measurement made on one system and the values ascribed to a space-like separated system [38, 39, 40]. Even so, it is of interest to consider the relation of the famous incompleteness argument made by Einstein, Podolsky and Rosen (EPR) [18] to the principle of complementarity, as embodied in Eq. (40).

The EPR paper considers two particles described by an eigenket of relative position and total momentum [18]. Clearly, the position of the first particle can be estimated precisely by 
a direct measurement of the position, with perfect accuracy: $\epsilon\left(\mathcal{X}_{\text {opt }}\right)=0$. Simultaneously, the correlation between the particles allows the momentum of the first particle to also be estimated precisely, from a measurement of the momentum of the second particle, again with perfect accuracy: $\epsilon\left(\mathcal{P}_{\text {opt }}\right)=0$. At first sight it thus appears that the universal jointmeasurement uncertainty relation in Eq. (40) is violated by the EPR example.

To see what is happening, it is helpful to replace the non-normalisable eigenket considered by EPR with the physical wavefunction

$$
\psi\left(x, x^{\prime}\right)=K e^{-\left(x-x^{\prime}-a\right)^{2} / 4 \sigma^{2}} e^{-\tau^{2}\left(x+x^{\prime}\right)^{2} / 4 \hbar^{2}} e^{i p_{0}\left(x+x^{\prime}\right) / 2 \hbar},
$$

where $K$ is a normalisation constant and $\sigma, \tau \ll 1$ in suitable units. One has

$$
\begin{aligned}
& \left\langle X-X^{\prime}\right\rangle=a, \quad \operatorname{Var}\left(X-X^{\prime}\right)=\sigma^{2} \ll 1, \\
& \left\langle P+P^{\prime}\right\rangle=p_{0}, \quad \operatorname{Var}\left(P+P^{\prime}\right)=\tau^{2} \ll 1,
\end{aligned}
$$

and hence $\psi$ is an approximate eigenstate of the relative position and total momentum.

Suppose now that $X$ and $P^{\prime}$ are simultaneously measured as before, with measurement results $x$ and $p^{\prime}$ respectively. The corresponding best possible estimates of $X$ and $P$ then follow via Eq. (8) as

$$
\tilde{X}_{\mathrm{opt}}=x, \quad \tilde{P}_{\mathrm{opt}}=\frac{\hbar^{2}\left(p_{0}-p^{\prime}\right)+\sigma^{2} \tau^{2} p^{\prime}}{\hbar^{2}+\sigma^{2} \tau^{2}} \approx p_{0}-p^{\prime}
$$

The dispersions and inaccuracies of these estimates follow from straightforward calculation as

$$
\begin{gathered}
\Delta \mathcal{X}_{\mathrm{opt}}=\left(\hbar^{2}+\sigma^{2} \tau^{2}\right)^{1 / 2} /(2 \tau) \approx \hbar /(2 \tau), \quad \epsilon\left(\mathcal{X}_{\mathrm{opt}}\right)=0, \\
\Delta \mathcal{P}_{\mathrm{opt}}=\frac{\left|\hbar^{2}-\sigma^{2} \tau^{2}\right|}{2 \sigma\left(\hbar^{2}+\sigma^{2} \tau^{2}\right)^{1 / 2}} \approx \hbar /(2 \sigma), \quad \epsilon\left(\mathcal{P}_{\mathrm{opt}}\right)=\frac{\hbar \tau}{\left(\hbar^{2}+\sigma^{2} \tau^{2}\right)^{1 / 2}} \approx \tau .
\end{gathered}
$$

Substitution into the lefthand side of the joint measurement uncertainty relation in Eq. (40) then gives $\hbar / 2$, which is precisely equal to the value of the righthand side - the state is in fact a minimum joint-uncertainty state of position and momentum (other equalities for this state are given in Ref. [26], where the effect of wavefunction collapse on optimal estimates is also considered).

The above results support, in a quantitative manner, Bohr's defence of the consistency of complementarity with the completeness of the standard quantum formalism [10, 19]. The EPR argument in fact goes somewhat further, asserting the physical reality of the 
estimated value of $P$ from the measurement of $P^{\prime}$, and the simultaneous physical reality of the estimated value of $X$ following from the alternative measurement of $X^{\prime}$ [18]. However, precisely because these measurements do not refer to a single experimental setup, such assertions go beyond the quantum formalism, and cannot be tested via Eq. (40).

More generally, even when one has full knowledge of the state of some system, and uses this prior information to make the best possible estimates of two complementary observables from a given experimental setup, there remains a fundamental tradeoff between dispersion and inaccuracy - embodied by the universal uncertainty relation in Eq. (40) - which prevents simultaneous knowledge of the corresponding physical properties.

\section{Example: linear estimates}

It is of interest to consider an example where one does not know the state of the system before measurement, but does have prior knowledge of the averages of one or more observables. While such prior information is by itself insufficient to make an optimal estimate as per Eq. (10), it can still be taken into account to improve on the "no information" estimate of Eq. (13).

One method of proceeding might be to introduce some physical principle to assign a unique state to the system that is consistent with the given prior information, and to calculate estimates by substituting this state for $\rho$ in Eq. (10). For example, the maximum entropy principle of Jaynes could be used for this purpose [41] (indeed the "thermodynamic" example in Eq. (15) may be reinterpreted in this way, where the form of the density operator corresponds to the maximum entropy state consistent with a known prior average energy of the system [41]).

In general, however, there are many possible physical states consistent with given prior knowledge about certain averages. Further, the available prior information may well imply, for example, that the system is not described by a maximum entropy state (eg, in a communication setup it may be known that each signal is described by one of a number of

fixed pure states $\left|\psi_{1}\right\rangle,\left|\psi_{2}\right\rangle, \ldots$ having equal average energies). It is therefore important to consider estimation methods that use only the prior information that is available, without requiring assumptions about the actual state of the system. Here linear estimates and their joint uncertainty properties will be examined. 
Consider first a detection system for a classical signal $s$, which is subject to uncorrelated noise $n$, resulting in a measured signal $m=s+n$. It will be assumed that $\langle n\rangle=0$. If $m$ is taken as an estimate for $s$, the average deviation of this estimate from the actual signal $s$ is quantified by

$$
\epsilon^{2}=\left\langle(m-s)^{2}\right\rangle=N
$$

where $N$ denotes the noise variance $\left\langle n^{2}\right\rangle$.

However, one can do better if there is some prior information about the signal statistics. For example, suppose one knows the average value $\bar{s}=\langle s\rangle$ and the variance $S=\left\langle(s-\bar{s})^{2}\right\rangle$ of the signal. Then it is straightforward to show that the linear estimate $m_{\text {lin }}=\lambda m+(1-\lambda) \bar{s}$ has a minimum statistical deviation from the signal $s$ given by

$$
\epsilon_{\text {lin }}^{2}=\left\langle\left(m_{\text {lin }}-s\right)^{2}\right\rangle=N S /(S+N)<\epsilon^{2}
$$

corresponding to the choice $\lambda=S /(S+N)$. The associated rms uncertainty of this estimate follows as

$$
\Delta m_{\text {lin }}=S /(S+N)^{1 / 2}=(1+N / S)^{-1} \Delta m
$$

Thus, use of the prior information allows not only a better estimate of the signal, but also a reduction in the dispersion of the estimate of the signal. Note that for the particular case of Gaussian signal and noise distributions, the above linear estimate is in fact optimal over any other estimate [42].

Consider now the canonical joint measurement of position and momentum for a quantum particle as previously discussed, corresponding to measurement of the commuting operators $X_{J}=X+X^{\prime}, P_{J}=P-P^{\prime}$, where the primed variables refer to an auxilary particle in a minimum uncertainty state with $\left\langle X^{\prime}\right\rangle=\left\langle P^{\prime}\right\rangle=0$. It will be assumed that all that is known about the particle prior to measurement are the means and variances of $X$ and $P$.

The observables $X, X^{\prime}, X_{J}=X+X^{\prime}$ all commute, and are therefore completely analogous to the respective classical variables $s, n$, and $m=s+n$. It immediately follows that the best linear estimate of $X$ from $X_{J}$, given knowledge of $\langle X\rangle$ and $\operatorname{Var} X$, is equivalent to measurement of the operator $X_{\text {lin }}=\lambda X_{J}+(1-\lambda)\langle X\rangle$, with $\lambda=\left(1+\operatorname{Var} X^{\prime} / \operatorname{Var} X\right)^{-1}$; associated inaccuracy

$$
\epsilon\left(X_{\text {lin }}\right)=\Delta X \Delta X^{\prime} /\left(\operatorname{Var} X+\operatorname{Var} X^{\prime}\right)^{1 / 2}
$$


and associated dispersion

$$
\Delta X_{\text {lin }}=\operatorname{Var} X /\left(\operatorname{Var} X+\operatorname{Var} X^{\prime}\right)^{1 / 2}=\left(1+\operatorname{Var} X^{\prime} / \operatorname{Var} X\right)^{-1} \Delta X_{J}
$$

One similarly has an optimal linear estimate $P_{\text {lin }}$ obtained from knowledge of $\langle P\rangle$ and $\operatorname{Var} P$, with analogous expressions for $\epsilon\left(P_{\text {lin }}\right)$ and $\Delta P_{\text {lin }}$.

Note that there is a degree of freedom remaining, which may be tuned for further optimality. In particular, the squeezing ratio $\Delta X^{\prime} / \Delta P^{\prime}$ may be chosen to minimise some suitable cost function. For example, for a harmonic oscillator one might choose to minimise the "inaccuracy energy" $\epsilon\left(P_{\text {lin }}\right)^{2} /(2 m)+\left(m \omega^{2} / 2\right) \epsilon\left(X_{\text {lin }}\right)^{2}$. However, the existence of the universal uncertainty relation in Eq. (40) suggests the more generic "joint uncertainty" cost function

$$
J=\Delta X_{\text {lin }} \epsilon\left(P_{\text {lin }}\right)+\epsilon\left(X_{\text {lin }}\right) \Delta P_{\text {lin }}+\epsilon\left(X_{\text {lin }}\right) \epsilon\left(P_{\text {lin }}\right) .
$$

Minimising $J$ with respect to the squeezing ratio leads to two regimes. First, if $\Delta X \Delta P \leq$ $2 \hbar$, then it is optimal to choose $\Delta X^{\prime} / \Delta P^{\prime}=\Delta X / \Delta P$, which leads to the inequality

$$
\Delta X_{\text {lin }} \Delta P_{\text {lin }} \geq\left[1+\hbar^{2} /(4 \operatorname{Var} X \operatorname{Var} P)\right]^{-1} \hbar / 2 \geq \hbar / 4
$$

analogous to the lower bound in Eq. (35). However, for $\Delta X \Delta P>2 \hbar$, it is optimal to choose either of $\Delta X^{\prime}$ and $\Delta P^{\prime}$ equal to zero, corresponding to the alternatives $X_{\text {lin }}=X, P_{\text {lin }}=\langle P\rangle$ and $X_{\text {lin }}=\langle X\rangle, P_{\text {lin }}=P$ respectively - i.e., not to bother with a true joint measurement at all! A similar dichotomy of regimes has been noted previously for the special case of Gaussian states [43].

\section{CONCLUSIONS}

A general formula for the best possible estimate of one observable from the measurement of another has been given, and applied in a number of settings. A universal jointmeasurement uncertainty relation has also been given, which quantifies the principle of quantum complementarity for all possible experimental setups. Describing measurements by completely general POMs (which require only that probabilities are positive and sum to unity), implies that the main results of the paper are universally applicable, and independent of any dynamical models and interpretational issues concerning quantum measurement. It is also worth noting that the use of a general POM includes the case where an experimenter bases an estimate on the results of a plurality of measurements, obtained by carrying 
out a number of (predetermined) consecutive physical operations (described by "completely positive" linear maps [25]).

It has been shown that by using prior information about the system (eg, the state of the system in Sec. III.C and the mean and variance of certain observables in Sec. IV.C) one can improve the standard uncertainty relation for the canonical joint measurement of position and momentum by up to a factor of 4 . However, unlike the classical case, if one makes optimal use of complete information about the system before measurement, one cannot do any better than this - complementarity cannot be circumvented by the use of prior knowledge. The principle of complementarity is similarly consistent with respect to the properties of entangled systems - as demonstrated in Sec. IV.B, quantum correlations cannot be exploited to violate the universal joint-measurement uncertainty relation of Eq. (40).

Finally, it would be of interest to determine the best possible estimate of an observable under the imposition of further natural restrictions. For example, one could require that an estimate of photon number, from some general measurement, minimise statistical deviation subject to the further constraint of being a positive integer. This would reduce the accuracy of the estimate relative to the unconstrained case, but has the advantage of incorporating prior information about the possible physical values of the observable being estimated. It would similarly be of interest to consider alternative characterisations of dispersion and inaccuracy (eg, entropy and relative entropy).

Some time after this paper was submitted, a related eprint by Ozawa has appeared [45], giving an independent derivation of the universal uncertainty relation in Eq. (40).

\section{APPENDIX A}

The proofs of Eqs. (10) and (13), for optimal estimates of a Hermitian operator $A$ from a general measurement $\mathcal{M}$, are given here. The generalisation to the optimal estimate of any POM observable $\mathcal{A}$ from measurement of $\mathcal{M}$ is also discussed.

The main ingredient required is a measure of "how good" a given estimate of $A$ is. For the case of two Hermitian operators $A$ and $B$, a natural measure of how well one mimics the other, for a given state $\rho$, is given by the statistical deviation

$$
D_{\rho}(A, B)^{2}=\operatorname{tr}\left[\rho(A-B)^{2}\right]
$$


This measure was used in the proof of Eq. (8) for the special case where $\mathcal{M}$ corresponds to a Hermitian operator $M$. However, to consider arbitrary measurements $\mathcal{M}$ it is necessary to generalise this measure to the case where one observable is an arbitrary POM observable.

Fortunately, the generalisation of Eq. (A1) is quite straightforward [9, 32]. In particular, it is natural to define the statistical deviation between a Hermitian operator $A$ and a POM observable $\mathcal{M}=\left\{M_{m}\right\}$ by

$$
D_{\rho}(A, \mathcal{M})^{2}=\sum_{m} \operatorname{tr}\left[M_{m}(A-m) \rho(A-m)\right]=\operatorname{tr}\left[\rho(A-\bar{M})^{2}\right]+\operatorname{tr}\left[\rho\left(\overline{M^{2}}-\bar{M}^{2}\right)\right],
$$

where $\overline{M^{j}}:=\sum_{m} m^{j} M_{m}$. This expression reduces to Eq. (A1) for Hermitian observables. It follows directly from a natural algebra for POM observables [32] (being the square root of the average of the square of the "difference" of two such obervables), and has also been postulated ab initio in Ref. [9]. It first appeared in the context of estimation of photon number from an optical phase measurement [44].

To obtain Eq. (10), let $\mathcal{A}_{f}$ denote the observable corresponding to a general estimate of $A$ from $\mathcal{M}$, where outcome $m$ of $\mathcal{M}$ corresponds to outcome $f(m)$ of $\mathcal{A}_{f}$. The statistical deviation between $\mathcal{A}_{f}$ and $A$ follows from the first equality in Eq. (A2) as

$$
\begin{aligned}
D\left(A, \mathcal{A}_{f}\right)^{2} & =\left\langle A^{2}\right\rangle-\sum_{m} f(m) \operatorname{tr}\left[\rho\left(A M_{m}+M_{m} A\right)\right]+\sum_{m} f(m)^{2} \operatorname{tr}\left[\rho M_{m}\right] \\
& =\left\langle A^{2}\right\rangle-2 \sum_{m} f(m) \tilde{A}_{\mathrm{opt}}(m \mid \rho) \operatorname{tr}\left[\rho M_{m}\right]+\sum_{m} f(m)^{2} \operatorname{tr}\left[\rho M_{m}\right] \\
& =\left\langle A^{2}\right\rangle-\sum_{m} \tilde{A}_{\mathrm{opt}}(m \mid \rho)^{2} \operatorname{tr}\left[\rho M_{m}\right]+\sum_{m}\left[f(m)-\tilde{A}_{\mathrm{opt}}(m \mid \rho)\right]^{2} \operatorname{tr}\left[\rho M_{m}\right],
\end{aligned}
$$

where $\tilde{A}_{\text {opt }}(m \mid \rho)$ is the estimate defined in Eq. (10). The last term is nonnegative, and hence the statistical deviation is minimised by the choice $f(m)=\tilde{A}_{\text {opt }}(m \mid \rho)$, as per Eq. (10). Note that choosing $\mathcal{A}_{f}=\mathcal{A}_{\mathrm{opt}}$ in the above expression, and using Eq. (11), gives

$$
D_{\rho}\left(A, \mathcal{A}_{\mathrm{opt}}\right)^{2}=\left\langle A^{2}\right\rangle-\left\langle\mathcal{A}_{\mathrm{opt}}^{2}\right\rangle=\operatorname{Var} A-\operatorname{Var} \mathcal{A}_{\mathrm{opt}}=D_{\rho}(A,\langle A\rangle)^{2}-D_{\rho}\left(\mathcal{A}_{\mathrm{opt}},\langle A\rangle\right)^{2}
$$

for the minimum statistical deviation.

The proof of Eq. (13) is completely analogous, where the statistical deviation in Eq. (A2) is replaced by the generalised Hilbert-Schmidt distance

$$
\begin{aligned}
d(A, \mathcal{M})^{2} & :=\sum_{m} \operatorname{tr}\left[M_{m}(A-m)^{2}\right] \\
& =\operatorname{tr}\left[(A-\bar{M})^{2}\right]+\operatorname{tr}\left[\left(\overline{M^{2}}-\bar{M}^{2}\right)\right]
\end{aligned}
$$


obtained via a natural algebra for POM observables [32]. Note that this measure is proportional to the average of the square of the statistical deviation over all states.

Finally, it may be asked whether one can define the best possible estimate when $\mathcal{A}$ does not correspond to a Hermitian operator. This is of interest, for example, if one wants to make the best estimate of elapsed time or optical phase from the measurement of some observable such as position or photon number. It turns out that the generalisation of statistical deviation is highly non-trivial in this case, as certain consistency conditions must be satisfied [32]. However, for the special case of complete observables $\mathcal{A}$ and $\mathcal{M}$ (i.e., with $A_{a}=|a\rangle\langle a|$, $\left.M_{m}=|m\rangle\langle m|\right)$, which further satisfy the condition that no two kets from the combined set $\{|a\rangle,|m\rangle\}$ are proportional, it follows from Sec. 4 of [32] that the statistical deviation has the simple generalised form

$$
D_{\rho}(\mathcal{A}, \mathcal{M})^{2}=\operatorname{tr}\left[\rho\left(\overline{A^{2}}+\overline{M^{2}}-\bar{A} \bar{M}-\bar{M} \bar{A}\right)\right]
$$

with $\overline{A^{j}}$ and $\overline{M^{j}}$ defined as above. It may be shown that the best possible estimate of $\mathcal{A}$, from a measurement result $m$ of $\mathcal{M}$ on a known state $\rho$, follows in this case as

$$
\tilde{A}_{\text {opt }}(m \mid \rho)=\frac{\langle m|\rho \bar{A}+\bar{A} \rho| m\rangle}{2\langle m|\rho| m\rangle} .
$$

However, more generally one cannot simply replace $A$ by $\bar{A}$ in Eq. (10).

[1] C.W. Helstrom, Quantum Detection and Estimation Theory (Academic Press, New York, 1976).

[2] A.S. Holevo, Probabilistic and Statistical Aspects of Quantum Theory (North-Holland, Amsterdam, 1982).

[3] S.L. Braunstein, C.M. Caves, and G.J. Milburn, Ann. Phys. (N.Y.) 247, 135 (1996).

[4] W. Heisenberg, The Physical Principles of the Quantum Theory (Dover, USA, 1930). The quote is from page 20 .

[5] Y. Aharonov and D. Bohm, Phys. Rev. 122, 1649 (1961).

[6] D.M. Appleby, Int. J. Theor. Phys. 37, 1491 (1998).

[7] G.M. D'Ariano, quant-ph/0208110.

[8] M. Ozawa, Phys. Rev. A 67, 042105 (2003). 
[9] M. Ozawa, quant-ph/0307057.

[10] N. Bohr, Atomic Physics and Human Knowledge (Wiley, New York, 1958), pp. 32-66.

[11] E. Arthurs and J.L. Kelly, Jr., Bell Syst. Tech. J. 44, 725 (1965).

[12] W.K. Wootters and W.H. Zurek, Phys. Rev. D 19, 473 (1979).

[13] E. Arthurs and M.S. Goodman, Phys. Rev. Lett. 60, 2447 (1988).

[14] H. Martens and W. de Muynck, Found. Phys. 20, 255 (1990).

[15] G. Jaeger, A. Shimony, and L. Vaidman, Phys. Rev. A 51, 54 (1995).

[16] W.M. de Muynck, Found. Phys. 30, 205 (2000).

[17] A. Trifonov, G. Björk, and J. Söderholm, Phys. Rev. Lett. 86, 4423 (2001).

[18] A. Einstein, B. Podolsky, and N. Rosen, Phys. Rev. 47, 777 (1935).

[19] N. Bohr, Phys. Rev. 48, 696 (1935).

[20] K.K. Wan and P.J. Sumner, Phys. Lett. A 128, 458 (1988).

[21] P.R. Holland, The Quantum Theory of Motion (Cambridge University Press, UK, 1993), chapter 3 .

[22] L. Cohen, Phys. Lett. A 212, 315 (1996).

[23] Y. Aharonov, D.Z. Albert, and L. Vaidman, Phys. Rev. Lett. 60, 1351 (1988).

[24] Y. Aharonov and L. Vaidman, Phys. Rev. A 41, 11 (1990).

[25] P. Busch, M. Grabowski, and P.J. Lahti, Operational Quantum Physics (Springer, Berlin, 1995), chapters II, VII.

[26] M.J.W. Hall, Phys. Rev. A 64, 052103 (2001).

[27] H.M. Wiseman, Phys. Rev. A 65, 032111 (2002).

[28] L.M. Johansen, quant-ph/0308137.

[29] M.J.W. Hall, Phys. Rev. A 62, 012107 (2000).

[30] A. Luis, Phys. Rev. A 67, 064101 (2003).

[31] Two compatible (eg, classical) observables $X$ and $Y$ will always have some joint probability distribution $p(x, y)$. Minimising the statistical deviation $\left\langle[X-f(Y)]^{2}\right\rangle$ then leads to the best possible estimate $f(y)=\int d x x p(x, y) / \int d x p(x, y)$ for $X$ from measurement result $Y=y$, analogous to Eq. (10) [26, 30]. Clearly this is always nonnegative for a positive observable $X$.

[32] M.J.W. Hall, quant-ph/0302007.

[33] H.P. Yuen and J.H. Shapiro, IEEE Trans. Inf. Theory IT-26, 78 (1980).

[34] J.H. Shapiro and S.S. Wagner, IEEE J. Quantum Electron. QE-20, 803, (1984). 
[35] C.M. Caves and P.D. Drummond, Rev. Mod. Phys. 66, 481 (1994).

[36] M.J.W. Hall and I.G. Fuss, Quantum Opt. 3, 147 (1991).

[37] A. Dembo, T.M. Cover, and J.A. Thomas, IEEE Trans. Inf. Theory IT-37, 1501 (1991).

[38] J.S. Bell, Physics 1, 195 (1964).

[39] A. Shimony, M.A. Horne, and J.F. Clauser, Dialectica 39, 97 (1985).

[40] B. d'Espagnat, Veiled Reality, An Analysis of Present-Day Quantum Mechanical Concepts (Addison-Wesley, Reading, 1995), Appendix 4.

[41] E.T. Jaynes, Phys. Rev. 108, 171 (1957).

[42] J.C. Hancock and P.A. Wintz, Signal Detection Theory (McGraw-Hill, New York, 1966), pp. 128-130.

[43] A.S. Holevo, Statistical Structure of Quantum Theory, (Springer, Berlin, 2001), Sec. 2.2.4.

[44] M.J.W. Hall, quant-ph/0103072.

[45] M. Ozawa, quant-ph/0310070. 